\title{
The path towards validating Tibetan and Chinese medicine by the food and drug administration
}

Keywords: Tibetan medicine, US. healthcare system, complementary/alternative medicine, Germany's herb regulation system, Chinese medicine in America

\section{Introduction}

This paper aims to offer an overview of the history of Tibetan medicine and its emerging contributions to Western mainstream medical culture and to compare Tibetan medicine with Western medicine. Within this, there will be a brief introduction about the current status of Tibetan Medicine in the US; followed by a proposal to change federal drug and clinical policy guidelines on botanical drugs and alternative medicine applying German drug policies as exemplary guidance.

In recent years, the United State's increasing reliance on Tibetan health practitioners is evidenced by the public's interest in an alternative to conventional Western medicine. Tibetan medicine has been integrated into and has made large contributions to the U.S. Healthcare system and yet despite all of this recognition and support, Tibetan medicine remains unregulated by US Health and Drug agencies. Measures and guidelines, with exemplars from Germany's management of botanical medicines, will be used to evaluate how Tibetan medicine meets and exceeds federal standards of medical safety and protection. The results of which will contribute to the public's knowledge and increase incentives for ongoing research. The ultimate goal is to change the U.S States' attitude about Eastern medicines so much so that they gain regulation from the Federal Drug Administration.

Tibetan medicine is an ancient science with a history exceeding 2300 years of documented practice. Oral tradition, artifacts, and legends dating back to prehistoric eras serve as evidence. Contemporary Tibetan Medicine is based on the cumulative sum of the knowledge, skill and values of an unbroken lineage of ancestors. Through keen observation and empirical science these practitioners were committed to generating health practices that gendered long and productive lives-- always with the aim of the amelioration and elimination of the suffering associated with the inherent and emerging environmental conditions and human diseases. With this abiding devotion to the cessation of suffering and the causes of suffering, Tibetan medical scholars collaborated with, drew upon, and assimilated the "best practices" in medicine of their Chinese, Indian, Nepalese, Mongolian, Turkistan, (what is now) Pakistani, Arab, Persian, and Bhutanese medical colleagues.

The distinctive geography, geology and vegetation of the Tibetan plateau along with the cultural and scholarly traditions of its people gave rise to the development and testing of the unique pharmacological resources for utilization in medical treatments. Today's distinguished Tibetan medical system emerged gradually through the repeated practice and improvement in the science of understanding human physiology, pathology, diagnosis, drug preparations, and body treatments.

\author{
Volume II Issue 6 - 2018
}

\author{
Tashi Rabten \\ China University Political Science Law, China
}

Correspondence: Tashi Rabten, China University Political Science Law, PhD Candidate, 1 I 7 Ridge Road,Valley Cottage, NY 10989, USA, Email Rabtan@me.com Received: September 30, 2018 | Published: November 07,
2018

Tibetan medicine is a healing science (gso-ba rig-pa) and ancient medical system based on the Buddhist view of the universe in which everything is interrelated. Through thousands of years of observation and practice, Tibetan practitioners have developed multiple yet unique methods of understanding the structure of the internal organs and the human body's physiological and psychological processes. These early practitioners, in fact, may be considered among the first medical empirical scientists to develop a systematic body of knowledge through careful observation.

Tibetan medicine, as a healing science, focuses on the inseparability of the human body and mind. Mind and body, as one system, and how that impacts health; therefore, a healthy mind contributes to a healthy body and healthy bodies contribute to healthy minds. There is no psyche/soma duality. Tibetan medicine recognizes the two as one in human biology and treats - what is split in western science as nondual in cases of pathology.

The origins of Tibetan medical history are only briefly described here. While the provenance of Tibetan Medicine as cultural patrimony as it is debated by China and India in respective governmental applications to UNESCO).

Tibetan Medicine in the US is referred to as "Non-Western, Alternative, Complementary and even, Oriental Medicine" thereby limiting its scope of availability and practice.

The biggest hurdle Tibetan Medicine faces to becoming regulated is the politically contested nature of the U.S healthcare system. Debates persist on cost, access, eligibility, and coverage with myriad tiered limitations. No nation invests more per capita for health care than the United States. Medical specialists are disproportionately concentrated geographically and generally reserved for those with informed access and insurance. As a disease and symptom-specific model of medicine, US clinical research and technologies are notable yet within a global context, the cost of health care in the US increases exponentially on an annual basis and as such makes one wonder where the money is spent. US determinants of health and relevant treatment outcomes fail to meet standard measures of progress of other developed states. Well documented by national and international scholars, comparatively America ranks $20^{\text {th }}$ in infant mortality on the aggregate, $29^{\text {th }}$ in lowbirth-weight with greater disparities in under-resourced communities and, overall $6^{\text {th }}$ in life expectancy. 
Financial incentives remain at the core of the problem. The U.S Healthcare industry encourages reliance on costly technology in assessment, diagnosis, treatment, specializations, and pharmaceuticals. Despite the strengths (and weaknesses) of the US medical model, it is interesting to point out that increasingly consumers are seeking alternative care. Their preference is towards a more holistic, less costly, and less surgically invasive form of treatment.

Today, US health care commands over $20 \%$ of the Gross Domestic Product. Nearly one-quarter of Americans rely on complementary alternative care at a cost of 1percent of the aggregate health care budget .A comparative analysis of costs of western allopathic medicine with alternative nonwestern treatments is not the focus of this commentary, however, it is indisputable that complementary care costs are significantly lower with superior health outcomes, for pain management, cancer-related anorexia, joint and bone disease, rheumatoid arthritis, sciatica, hemiplegia, polio, postpartum distress, urticarial, neurological conditions such as Multiple Sclerosis, and other diseases in Third World countries.

\section{US regulation of health and pharmaceuticals}

Even though Healthcare per se is not mentioned in the US Constitution, within this document it establishes laws that confer certain rights to the national government and others to the individual states in regard to regulating and protecting the health, safety, and general welfare of its citizens.

To this point: The Medical Practice Act has been adopted by every state for the assurance and protection from the unprofessional and unlawful practice of medicine. These regulations govern most aspects concerning the practice of medicine while providing a safety mechanism between the medical profession and the general public.

Tibetan medicine is categorized as non-mainstream, Complementary/Alternative Medicine (CAM) in the US, which marginalizes it from conventional Western medicine and in so doing deprives many patients of its benefits. In general, the practice of Tibetan medicine with its herbal, dietary supplements, therapeutic technologies, meditation, Reiki, and unique understanding of human physiology are subsumed under CAM law.

\section{Historical overview of Tibetan medicine}

Tibetan peoples, from the dawn of human civilization, observed their natural habitat, its flora and fauna, to inform their healing practices. The first identified human"disease" was indigestion. Observing the self-care of animals combined with attention to human distress, early Tibetan medical practitioners documented that rough, raw food resulted in considerable discomfort and indigestion. Later people found the use of fire as a method to convert raw, cold foods to better suit human digestion. Fire (and heat) as an essential environmental element transformed nourishment for easier digestion, further, fire-heated foods not only prevented indigestion and "Bad" diseases from uncooked meats by improving overall gastro-intestinal health.

Early Tibetan healing practices discovered and utilized the knowledge that water increases body heat and facilitate healthy digestion. The essential natural elements of water and heat (fire) were also found beneficial in the treatment of other diseases, and in parallel historical periods contributed to the development of agriculture and animal husbandry.
Tibetan practitioners were also the inventors of many methods for healing traumatic injuries and wound care. They developed specific techniques of refining and extracting butter to treat trauma: "to stop bleeding, apply to sore[s]". They devised many other methods, including obstetrical postpartum indigestion to restore maternal vitality and topical application to heal peritoneal tearing. Keenly observant of the natural environment, there is a record of continuous discovery of new and organic botanical, as well as veterinary, sources for healing common ailments Tibetan ancestors, over millennia, formulated and built systems of knowledge in ten academic subjects. The five main traditional subjects include Architecture, Medicine, Linguistics, Logic, and Buddhist Philosophy; the other five, less renowned scholarly traditions, are in poetry, rhetoric, rhyme, drama, and astrology.

Tibetan medicine is a significant subject of scholarship that has evolved within the contexts of geography, culture and ideologies, owing much to the abiding resilience inherent among Tibetans and their collaboration with neighboring fraternal peoples like the Mongolians and the Bhutanese. Historically, Tibetan medical practitioners and scholars assimilated the results of collaboration in the sciences and medicine to advance, invent, improve, and create a unique and complete scientific medical system. Tibetan medical practices are now widely adopted by fraternal peoples and contribute to the wellbeing of indigenous groups throughout Asia.

\section{Principles of Tibetan medicine}

Tibetan medicine pays close attention to three factors essential to human physiological functions: Breath or "wind" or the element of Air summarized as "Long" (referring to wind); metabolic and endocrinological vitality or "red Tripa" (referring to the element to fire); and "Pekon" (referring to mucus).

\section{Long divided five longs}

The main function of the human body is to maintain life with vascular circulation, physical mobility, and decomposition and metabolism of food.

\section{Tripa}

Understanding bile as an essential function in the human body contributes to knowledge about the production and regulation of body temperature, vitality, energy, pallor, digestion, enzymatic fermentation, etc.

\section{Pekon liquid}

This liquid assesses of the balance of Pekon (or mucus generation) in the body and its role and function in nutrition, adjusting temperature, and cooling the body to achieve normal sleep patterns.

Tibetan medicine asserts, through empirical observation, practice, and research dating from the earliest period of human history, that when the above three factors function in the human body to support a core balance, there is normal life-sustaining physiological functioning. Alternatively, when out of balance, these essential human physiological functions produce a variety of diseases.

Through time-worn, age-tested research, observation, and practice, Tibetan medical texts affirm that the body is composed of seven substances, namely: the diet of tissue and blood, meat, fat, bone, marrow and life-sustaining essences, for example, semen. 
Seven Body Constituents are:

i. Essential nutrients from ingested foodstuffs

ii. Blood

iii. Muscle tissues

iv. Fatty tissues

v. Bone

vi. Marrow

vii. Regenerative Fluides

The human body as a whole is linked together by a meridian system that brings vital life supporting energy, blood and fluids to all its organs and tissues. One of the tenets of Tibetan medicine is the inter-relationship and the inseparability between people and nature. Consequently, when nature is out of balance or in crisis, people will experience a corresponding physiological effect.

Tibetan medical assessment and diagnosis methodologically applies the use of "look, touch, ask." As well as observation and attention to the pulse, [see original Four Medical Tantras text on "inch, off, feet"] the knowledge of the pulse and meridien connections to internal organs, and in particular, plays a very significant role of the diagnosis of "urine". It takes years for doctors to learn urinalysis techniques. When compared to modern diagnostic instruments, Tibetan doctors have been proven to be as equally accurate in their diagnoses.

In addition to the use of plants, animals, minerals and, Tibetan medical practices include the ancient medical arts and sciences of (acu) puncture, bleeding, moxabustion, meditation, yoga, healing mineral medicinal baths and other therapies.

An essential theory of Tibetan Buddhism assumes that three main poisons rule the existence of all beings, including humans. These three poisons are greed, delusion and sensual attachment. Furthermore, Tibetan Medicine interprets and assesses three elemental energies: Wind (tib: rLung), Bile (tib: mKhrispa, pronunciation :) and Phlegm (tib: Badken). These three constituent energies constantly flow in the human body and sustain health along with cognitive awareness. When these three constitutional energies are balanced at personal levels and well-being ensues. Alternatively, when confronted with internal cognitive distress or distraction from external stressors, the consequential loss of psychological and interpersonal balance potentiates illness. Therefore, practitioners ascribing to essential principles of Tibetan Medicine aspire to equally treat the mind and body's constituent energies.

\section{Core concepts of Tibetan medicine}

A. rLung is the source of the body's ability to circulate physical substances as well as the channel energy of the mind. In embryological development, neurological (mind) and physical (body) development is manifested as the system of rLung.

B. mKhris-pa is the key resource of metabolism in the production of heat, primary in the digestive system; mKhris-pa plays major roles in transformation of nutrients to produce life-sustaining qualities of blood.

C. Bad-kan is the source of the body's moisture, liquids and cold, often manifested in Chinese medicine as "yin." It maintains the body's cooling system, cross-system connectivity and stability. General Tibetan medical treatment methods include meditation as mental exercise; yoga for physical exercise; diet; herbal pharmaceuticals; and other therapeutic treatments-acupuncture; moxabustion; healing baths, etc.

\section{Tibetan medical ethics}

Tibetan medicine, in addition to its long history, holds and proscribes a very strong medical morality. Generally ascribing to the following ethical codes that meet and exceed the western Hippocratic code of "First, Do No Harm". ${ }^{1}$

First, all patients should be treated equally with dignity and respect: "[Treat] all the six sentient beings in [the] worlds of all beings, as [one's] parents" "love others better than [one] loves [oneself], whether enemies or friends, [do] not [be] hostile. ${ }^{2}$ Second, the doctor should have a noble quality - treatment should be accessible to all in need and not be expensive, especially when helping the poor; equality in treatment for both men and women, and "be there beauty or ugliness" it is ethically codified that practitioners should not be greedy, nor abhor female patients but greet them as their mother and sisters; avoid seeking personal gain, but always strive to avoid and deny impulses to be selfish, greedy, or cunning.

Third, the patient's condition must always be honored as confidential. The doctor should not be inexperienced [in the absence] of proper diagnosis or guess what disease [may be present]. He/she only offers diagnosis when and if there is an absolute grasp of the case. Only to the patient, may there be disclosure of the condition and situation of the disease. Further, patient education is emphasized; practitioners should inquire and dialogue with the patient about his or her understanding of the diagnosis and treatment as a method of assessing both doctor and patient comprehension and consent.

\section{Fourth, the doctor must be deeply committed to technical excellence:}

Yuthok Yonten Gonpo ${ }^{2}$ advised: when a practitioner still does not understand the patient's condition and attempts an operation [that may] "master" the patient's life away, this approach is despicable; where there is lack of knowledge and no relevant medical experience and the patient is given uninformed advice and explanation -- this is not a good doctor. ${ }^{2}$

The Tibetan medical oath in The Four Medical Tantras (rgyud bzhi) chapter three codifies that mutual respect between doctors is paramount: "[For] People receiving medical training, [they owe] their teachers great care and respect as [offered] to a god; between students, good relationships, mutual regard, mutual respect, mutual concern must be sustained."

\section{Overview of Tibetan medicine in Tibet}

Since the founding of the Peoples Republic of China (PRC) in 1949, a high degree of commercial attention focuses on Tibetan medicine. Also, India and China, as shown in their competing UNESCO applications (Ives, 2017) have well-established commercialized mass production of Tibetan pharmaceuticals. Tibetan medicine today brings its hosts a new era of profit and prosperity associated with the commoditization of Tibetan medicine. 
In 1916, Lhasa Medical College was established to disseminate medical care to the wider Tibetan community, and thereby removed non-standardized medical training from monasteries, temples and military camps.

In Lhasa, September, 1981, the Chinese Medical Association Society established the first modern conference on the international exchange of theoretical and clinical experiences. The conference received dozens of articles on Tibetan medical history, theory, clinical practice, various prescriptions, as well as the scientific research of experts and physicians.

In September 1991, the modern Tibetan Materia Medica codified the identification of raw herbs and more than 100 kinds of medicinal minerals (including plants, animals, minerals, etc.) with as many as 600 kinds of Tibetan treatments. Since the 1990s, Tibetan scholars have engaged in medical exchanges internationally. By 1993, the PRC regulation of standards and quality control in production emerged in the Central Ministry of Health Drug Standards Office. Through these measures, Tibetan medical efficacy has been demonstrated to be equivalent with modern scientific methods.

\section{Overview of Tibetan medicine in America}

News of Tibetan medicine arrived in the United States during the 1950's with the visit of Geshe Ngawang Wangyal. Later in the 1970's, His Holiness the Dalai Lama offered guidance on Tibetan Buddhism throughout the US, followed by the popular US guru, Chögyam Trungpa. Each of these significant figures emphasized the value of meditation and yoga in Tibetan Buddhism. Tibetan medicine was established in the US with the arrival of Dr. Yeshi Dhonden.

Now, Tibetan medicine has begun to be rigorously tested using Western scientific methodologies, but much more comparative research, experimentation, and study are warranted.

In the US, clinical trials have shown that Tibetan medicine effectively treats western clients. (For example, see: Dhondup \& Husted $^{3}$ on efficacy with conditions of Multiple Sclerosis.

Tibetan medicine, as a rule, focuses not only on the treatment of disease, but considers a patients' lifestyle, and the connection of cognition to behavior as core practices of this system. Therefore, yoga, Tai Chi, meditation, diet and exercise are collective means to maintain health.

New mixed scientific control and qualitative methodologies are needed to study Tibetan medicine as a holistic modality.

\section{Overview of Chinese medicine in America}

Chinese medicine was first introduced into the United States in the early 1800's when large numbers of Chinese immigrants came to seek a better life. With the advent of California's Gold Rush the number of immigrants and Chinese medicinal apothecaries soared.

American doctors at the time were so skeptical of these unknown ancient Eastern practices that they flat out rejected them, even going so far as to refer to them as "witch doctor science." The West has yet to recognize the East's approach to medicine as a legitimate practice; this is punctuated by the fact that the FDA has denied it legal status.

America's attitude towards Chinese medicine changed slightly during President Nixon's visit to China in 1971. During that trip,
New York Times journalist James B. Reston, who was documenting Mr. Nixon's trip, suffered an appendectomy. Mr. Reston was sent to a hospital and was administered acupuncture instead anesthesia to relieve his pain. He documented his experience in a New York Times article titled "Now, about my Operation in Peking".

Up until that point American's attitude towards acupuncture was that of a pseudo science. The article helped to educate the American public about acupunctures remarkable benefits and attributes. Suddenly, the ancient practice of acupuncture and Chinese herbal medicine was presented to mainstream America as a legitimate alternative to Western cures.

In the $21^{\text {th }}$ century, Traditional Chinese Medicine or TCM as it is categorized as "complementary/alternative medicine" by regulatory controls. TCM considers acupuncture as well as herbal and oriental body massage as legitimate form of healing. Except for acupuncture, none of TCM's therapies been regulated by the FDA, or the National Center for Complementary and Integrative Health. However, some herbs have some guidelines some supplements are useful in reducing the risk of certain diseases and are authorized to make label claims about these uses under the FDA as an approved dietary supplement. The number of acupuncture licenses as well as the needles that acupuncturists use are controlled by state regulation under Section 355 of the Federal Food, Drug, and Cosmetic Act, have soared.

TCM dietary supplements have numerous benefits and are generally considered to be a plant product or a nutritional hey have been proven to prevent diseases as such as Cardiovascular, Neurological Disorders, Muscular-skeletal, Respiratory System, Gastrointestinal, Gynecological and Psychological Disorders. They have also shown to improve the quality of life by activating normal bodily activities.

According to the Dietary Supplement Health and Education Act (DSHEA) passed by the U.S. Congress in December 1994, most natural medicines such as vitamins, minerals and botanicals are legally classified as "Dietary Supplements". Since these products do not require the FDA's drug review process, it is illegal to state the benefits of the medicine on the packaging and even goes so far as to state; "This product cannot be used for diagnosis, treatment and prevention of any disease".

According to the DSHEA regulations, a Dietary Supplement; the definition of a supplementary food is anything other than tobacco, which may contain one or more of the following dietary supplements: vitamins, minerals, plant based or botanical ingredients, amino acids, dietary supplements for increased absorption, concentrates, metabolites, individual ingredients and other various ingredients.

The DSHEA stipulates that these products can be directly labeled with nutritional benefits without the FDAs approval as long as they provide four types of nutritional functions:

A. Prevent and improve certain nutritional deficiencies and the epidemic diseases caused by them. An example can be seen with iodine and selenium that can improve and prevent Goiter and Keshan disease.

B. Describe some ingredients that may improve the structure and function of the human body. Such as calcium supplementation, which can improve the bodies, bone composition and increase its strength. 
C. Labeling the role and mechanism of components. For example, lecithin is a precursor of brain memory, and soy products contain a large amount of lecithin, which has been believed to enhance memory.

D. Certain ingredients can give overall comfort. Such as ginseng, and acanth panax,

Despite these many benefits, all bottles of herbal remedies must state, "These functions have not been evaluated by the FDA." Adding to the complications of giving Chinese medicine validity in the Western healthcare system is that under the law, if the FDA cancels the sale of a product it must provide clinical research data that it is unsafe for the product to be sold to the public. So, it is equally difficult to prove that a product is unsafe and that a product is safe.

Another challenge to the validity of TCM comes from the Dietary Supplement labeling committee that was appointed by President Clinton in 1997. This committee is composed of nutritionists, natural pharmacologists and lawyers who are responsible for product introductions, descriptions and regulatory management of drugs. Their prejudicial view is that since natural medicine comes from "nature" it has no validity at all.

For products that have sufficient scientific evidence to demonstrate their safety, are classified as Over-the-counter drugs or OTC, and those that do not yet have sufficient evidence (such as most Chinese medicines) are referred to as "food supplements". They argue that consumers have the right to obtain detailed research and literature before purchasing such products. That is why the FDA requires that manufacturers provide nutrition and functions related to such products.

In recent years, the American public has gradually realized that Chinese medicinal practitioners are competent and in some cases more desirable than Western doctors and treatments. Chinese medicine has changed from being treated with contempt and suspicion to gaining positive attention. Established research institutions, most of which are attached to comprehensive medical research units have also begun to pay more attention to TCM.

\section{There are currently about I46 TCM research institutions in the United States}

\section{The main ones are}

National Center of Complementary and Alternative Medicine (NCCAM); The National Institutes of Health (NIH), established in 1998, established office of Alternative Medicine (OAM), in 1992. The purpose of NIH is to promote the scientific evaluation of unconventional medicine through in-depth research, clarify its efficacy and to integrate it with modern medicine. The desired result is to gradually establish its legal status in the United States. NCCAM has a wide range of research, including traditional therapies in various countries. NCCAM provides a certain amount of sponsorship to traditional medical research all over the world every year, NCCA especially to traditional Chinese medicine. Among the projects it supports are acupuncture, Chinese medicine, tai chi, qigong, and massage. NCCAM also works with other government agencies such as the Health Protection Finance Division (HCFA), the Health Protection Policy Research Unit (AHCPR), and the Food and Drug Administration (FDA). Such cooperation facilitates the reassessment of existing regulations, which govern the research and use of traditional therapies in various countries (California Acupuncture Committee, American Institute of Chinese Medicine and Acupuncture Education Center).

Today, most states in the US have licensed and registered acupuncturists. However, the requirements of acupuncturists vary greatly from state to state. For example, New York State doctors have to undergo 300 hours of training, and non-doctors have to study for 3years to use acupuncture.

\section{Germany's herb regulation system}

The German drug management and health agency has defined "Botanicals" as:

"Different from chemicals, drugs containing preparations extracted from medicinal plants."

Botanicals can be sold in Germany in the form of drops, tablets, capsules or traditional herbal teas. Since Germany regards botanicals as common medicines, therefore the same management laws as other common chemicals apply. The German Drug Law stipulates that the listing of botanicals is subject to the approval of the Federal Agency for Drugs and Medical Devices (BfArM), just like chemicals. Compared to the policies in the United States with regard to validating and regulating Alternative Medicines, this radically different approach.

The German Federal Agency for Drugs and Medical Devices approves a drug based on its efficacy, safety and product quality. However, these "recognized" characteristics must be supported by proven data before they can be approved for marketing.

Alternately, it can be other "scientific knowledge/data" such as monographs, empirical reports or classic literature. Interestingly, the following three types of products have the same characteristics as botanicals, yet the German government does not classify them as botanicals:

The criteria are: Pharmacological, Toxicology and Clinical Research.

A. Products where a single component is isolated from plant extracts. This pure single ingredient extracted from plants, such as the ocean, Rehmannia, atropine, morphine, are not considered botanicals.

B. Products that contain food additives, and supplements, and food however, it hasn't mentioned that Chinese medicine has healing and treatment power instated of as food.

C. Products that contain a dose of natural drugs used in homeopathy and induction therapy.

According to the German Drug Law, the above three products do not need to go through the market approval process, nor do they need to meet the safety and quality standards for drug requirements. It must be noted that the above products are mainly used for nutrition and consumption, and they must comply with the relevant requirements of the German Food and Consumer Products Act (LMBG). Some foods can also be labeled with the words "helps to maintain the intestinal flora, and enhance the body." This allows certain vitamins, minerals, and plant products containing medical effects to be classified as medicines or foods. For example, mint tea can be sold either as a botanical or refreshing food, according to the trademark instructions. 


\section{Market overview of botanicals in Germany}

A healthy and natural life has become a common pursuit to people all over the world. In this international environment, more and more Germans are beginning to accept plant medicines into their lives as both legitimate cures and healthy additives. They feel this way, in part, because most drugs, supplements made by plants have limited to no side effects.

Plant medicines occupy a considerable share of the German pharmaceutical market. In particular, the share of the over-thecounter drug market has increased year by year. According to the EU's estimates, The EU's demand for botanicals is growing at an average annual rate of $10 \%$. According to a survey conducted by the Allensbach polling company in Germany in 2002, $73 \%$ of Germans currently use botanicals, of which women account for $79 \%$ of this usage.

People use plant medicines, mainly for the treatment of coughs, colds, stomach pains, indigestion, motion sickness, cardiovascular disease, anti-aging, and fatigue. Needless to say, people in Germany are paying more and more attention to ensuring their health through the use of botanicals.

The German government stipulates that only botanicals sold at pharmacies must be approved for marketing approval from the Federal Drug and Medical Device Administration. According to the data issued by the Federal Drug and Medical Device Administration, there are currently more than 1200 new approved plant agents in the German market. These include: hypericum leaves, ginkgo leaves, valerian roots, mountain lentils, chamomiles, and meddlers. Echinacea, garlic, and ginseng, and more than 3,700 traditional botanicals are in the process of being approved.

In 2013, sales of non-prescription drugs in German pharmacies were 9.8 billion Euros, of which botanicals accounted for $30 \%$, or about 3.07 billion euros. This number is conservative, as most beauty (pharmaceutical), health food stores, and even supermarkets in Germany sell botanicals.

German Management of Compensation for Botanical Drug Sales Channels and "National Medical Insurance" system. The German Drug Law states, "In principle, all medicines should be sold at pharmacies "However, it does not require botanicals to be sold with a doctor's prescription. Under certain conditions, medicines can also be sold outside the pharmacy through retail channels. For instance, they can be sold in retail channels such as Kräuter Kühne, LandVerte BioMarkt, Herbathek.

German Botanicals defend against diseases or those that can treat minor health problems such as Respiratory infections, cardiovascular disease and gastrointestinal problems. The German Drug Law also stipulates that drugs sold at retail outlets must be staffed with relevant and qualified medical professionals. Prescription botanicals can only be purchased at the pharmacy with a doctor's prescription. There are very few types of prescription botanicals prescribed by German law. The relevant legislature only listed certain botanicals as prescription drugs after receiving the advice of experts.

According to the German Social Code, in principle, over-thecounter botanicals purchased at pharmacies can also receive state compensation as prescription drugs. However, in reality, the amount of state-compensated botanicals purchased by the state is less than that of prescription drugs.

In 1994, the "German Federal Agency for Drugs and Medical Devices" replaced the "Federal Ministry of Health" as the German agency responsible for the safety, quality and efficacy evaluation and listing approval of pharmaceuticals and medical devices.

Some exceptions to this are made in section 36 of the German Drugs Act. If specified, a drug or group of drugs may be exempt from the listing approval process if it does not pose a risk to human or animal health. However, in order to ensure the quality, safety and efficacy of these products, such products must comply with the quality, efficacy and safety standards required by the German Federal Ministry of Health regarding approved products. These standards include test requirements, allowed product marks, and instructions on the package. Standers for quality control through reviewing the products safety and efficacy and registered to Germany. It is also documents in traditional and scientific approval That is to say, if the German federal government has approved the listing of botanical drugs and published its approval, it can be sold.

If the plant material of a drug is from a third party (a country outside the EU) according to the relevant regulations of Germany, such plants are regarded as "new chemical products" and must provide "scientific proof" of complete quality, safety and efficacy, especially its toxicity data and clinical records. However, oddly enough, there are no regulations in Germany for botanical raw materials from third countries.

\section{Germany's regulations on labeling and advertising of plant drugs}

In 1992, the EU issued Directive 92/27/EEC, which made uniform provisions for the labeling and packaging of human drugs, and required member states to implement the directives in their national laws. In 1994, Germany wrote the above-mentioned EU Directive into the German Medicines Act, and stipulated that the labeling and packaging regulations for botanicals were consistent with other chemicals. It is stipulated that for newly approved products, there must be a reminder of consumption information within one year after re-registration must accompany traditional botanical drugs. If the product does not have an outer packaging, the consumer information prompt must be indicated on the product label.

The EU Directive 92/28/EEC provides uniform regulations for pharmaceutical advertising. Germany also wrote this provision in the German Medicines Act in 1994. It is stipulated that, like other drugs, over-the-counter botanicals can be advertised. However, the German Food and Consumer Goods Act (LMBG) stipulate that food advertisements cannot expressly suggest that they have a medical effect. Therefore, German law does not allow the concept of "health products"

\section{Market importance of herbal medicines in Germany}

Herbs are over-the-counter medicines, such as birch leaves, golden rod and orthosiphon leaves but can be prescribed by physicians or practitioners for insurance reimbursement. In Germany $20 \%$ of herbs were sold as prescriptions and $80 \%$ were sold as over the counter medicines. 
Most importantly is that Germany has recognized herbs as a medicine and given market authorization for processing. In the Germany Medicines Act in 1994, emphasis was placed on active ingredients and not on individual products. This led to all herbs being carefully studied with monographs. The monographs are crucial to learn about an herbal medicine's safety and effectiveness. The monographs usually included Research findings, Botanical and common name(s), Contraindications, Dosage, Use, Action of the herb, and Historical use while also being focused on active ingredients and not on individual products. However, a Monograph is a way to document the herb; this process is crucial to safety and effective in marketing.

The Federal Institute for Drugs and Medical Devices, "Bundesinstitut für Arzneimittel und Medizinprodukte" (BfArM), is responsible for all herbal related products in regards to their safety and effectiveness. In addition, the Fifth Amendment of the German Medicines Act, states that some herbal medicine has been used "traditionally" and therefore Chinese and Tibetan medicine all belong to traditional medicine. This system authorizes herbal medicine's traditional usage and ensures that it can be traced and validated. This process does not include another scientific researcher and approval process on order to be marketed.

Alternatively, as an international exemplar, Germany, through the Federal Agency for Drugs and Medical Devices (BfArM) and the German Food and Consumer Products Act (LMBG), affirms the legitimacy of complementary medicine. Accordingly, health policy, efficacy trials, and reimbursement of consumer preference for phytopharmaceuticals in Germany, are conferred full legal status typically associated with medically prescribed allopathic pharmaceuticals. Botanical remedies are eligible for state reimbursement compensation, according to German Social Code. The German Drugs Act ensures the quality and standards of safety and efficacy of products with approval measures of testing, packaging, and consumer education. ${ }^{4} \mathrm{WHO}^{4}$ reports that nearly eight out of ten $(79 \%)$ of the German population rely on botanicals or herbal medicines and consider such remedies to be less harmful than commercially produced pharmaceuticals. Plant-based remedies command a significant share of the German drug market as consumers report more confidence in botanical efficacy associated with remedies arising from nature, with few or no side effects. ${ }^{4-6}$

It could be argued that given Tibetan Medicine's efficacy and major contribution to the study, treatment, and resolution of back pain, digestive disorders, headache, cancers, post-surgical conditions, and chemotherapies, that Tibetan Medicine justly falls under the auspice of state and federal Public Health Law; practitioner licensure laws; pharmaceutical approval. In the US, botanical pharmaceutical label laws for Tibetan medical prescriptions are best managed through the 1994 Dietary Supplement and Health Education Act (DSHEA) for quality, safety, and effect and not the Food and Drug Administration (FDA). Consumer movements in public health promote the Freedom of Choice in Health Care Act as another means of empowering complementary and alternative integrative medicine to expand into the accelerating market for holistic health care in the US. ${ }^{7-26}$

\section{Conclusion}

Tibetan medicine has helped people around the world for thousands of years. Tibetan medicine has also enhanced and advanced US "alternative medicine" policies. Seeing that Tibetan medicine is a much less costly and more efficient system of care than the conventional US medical system, it is in the best interest of the federal government and the individual states, and the nation as a whole to support Tibetan Medicine's ancient healing mission. Doing so would be a tremendous step towards the promotion of health and wellbeing for its citizens.

It is time that the United States adopted Germany's methods, regulations and attitudes toward Tibetan and Chinese herbal regulations. Aside from protecting and respecting the ancient practices of herbal medicines, it would also greatly benefit the United States and its citizens economically, psychologically and physically.

\section{Acknowledgements}

None.

\section{Conflict of interest}

Author declares that there is no conflict of interest.

\section{References}

1. Pavur, Claude. The Hippocratic Oath in Latin with English translation. 2013.

2. Yuthok Yonten Gonpo, Yu Tuo Yun Deng Gong Biography.

3. Dhondup L, Husted C. Tibetan Medical Interpretation of Myelin Lipids and Multiple Sclerosis. Ann N Y Acad Sci. 2009;1172:278-296.

4. WHO. Essential Medicines and Health Products Information Portal. Germany; 2017.

5. Busse R, Blümel M, Ognyanova D. Germany: Health System Review. Health Syst Transit. 2014;16(2):1-296.

6. Steinhoff B. The legal situation of phytomedicines in Germany. British Journal of Phytotherapy. 1993/94;3(2):76-80.

7. Alternative Medicine: The Healers. The Hopeful, And The Dingbats. By ACSH Staff. 2002.

8. Clarke TC, Black LI, Stussman BJ, et al. Trends in the use of complementary health approaches among adults: United States, 2002-2012. Natl Health Stat Report. 2015;79:1-16.

9. Commission on Dietary Supplement Labels. U.S. Government Printing Office. 1997.

10. Complementary and Alternative Medicine Products and Their regulation FDA. FDA. 2007

11. Dhonden Y. Exploring the Mysteries of Tibetan Medicine. New York Times; 1981.

12. Dhondup L, Husted C. Tibetan medicine and regeneration. Ann N Y Acad Sci. 2009;1172:115-122.

13. Egen O, Beatty K. Health and Social Conditions of the Poorest Versus Wealthiest Counties in the United States. Am J Public Health. 2017;107(1):130-135.

14. Four medical tantras (compiled in the 8th century, comprising four treatises: the Root Treatise, the Explanatory Treatise, the Practice Treatise, and the Appendices Treatise) can reasonably be taken as using basic Buddhist principles to reorganize all existing medical materials, including local Tibetan practices learnt from Indian and Chinese medicines. For information about the source of this classic, see Rinpoche (2001) pp. 3-4; Cai (2002) pp. 54-70 and Qiangbachilie (1996) pp. 1-18. For a Chinese translation, see Li (1983). 
15. Germany: Labeling and Marking Requirements. EU Single Marketing. 2018

16. Ives M. China and India File Rival Claims Over Tibetan Medicine. New York: Time. 2017

17. Jaya suriya DC. The regulation of medicinal plants - a preliminary review of selected aspects of national legislation. Unpublished Report.

18. Joos S, Glassen K, Musselman B. Herbal Medicine in Primary Healthcare in Germany: The Patient's Perspective. Evid Based Complement Alternat Med. 2012

19. Kieny MP, Bekedam H, Dolvo D, et al. Strengthening health systems for universal health coverage and sustainable development. Bull World Health Organ. 2017;95(7):537-539.

20. Marmot M, Bell R. The Sustainable Development Goals and Health Equity. Epidemiology. 2018;29(1):5-7.
21. Nahins RL. Expenditures on Complementary Health Approaches: United States, 2012. National Health Statistics Reports. 2016;95.

22. Pennyroyal G, Dhondup L, Husted C. A Review of Medicinal Plant Patents. Recent Patents on Biomedical Engineering. 2011;4:126-138.

23. Reston, James. Now, About My Operation in Peking. The New York Times. 1971.

24. Tomlinson T, Akerle O. Medicinal Plants: Their Role in Health and Biodiversity. Philadelphia: University of Pennsylvania Press; 1998. 240p.

25. Patricia Palma. Unexpected healers: Chinese medicine in the age of global migration (Lima and California, 1850-1930). História, Ciências, Saúde Manguinhos. 2018;25(1).

26. Zhang X. Regulatory Situation of Herbal Medicines A worldwide Review. Traditional Medicine Program. 1998. 